\title{
Research Article \\ Complexity of a Duopoly Game in the Electricity Market with Delayed Bounded Rationality
}

\author{
Junhai Ma and Hongliang Tu \\ College of Management and Economics, Tianjin University, Tianjin 300072, China \\ Correspondence should be addressed to Hongliang Tu, hltu1980@yahoo.cn
}

Received 2 May 2012; Accepted 21 November 2012

Academic Editor: Mingshu Peng

Copyright ( $) 2012$ J. Ma and H. Tu. This is an open access article distributed under the Creative Commons Attribution License, which permits unrestricted use, distribution, and reproduction in any medium, provided the original work is properly cited.

According to a triopoly game model in the electricity market with bounded rational players, a new Cournot duopoly game model with delayed bounded rationality is established. The model is closer to the reality of the electricity market and worth spreading in oligopoly. By using the theory of bifurcations of dynamical systems, local stable region of Nash equilibrium point is obtained. Its complex dynamics is demonstrated by means of the largest Lyapunov exponent, bifurcation diagrams, phase portraits, and fractal dimensions. Since the output adjustment speed parameters are varied, the stability of Nash equilibrium gives rise to complex dynamics such as cycles of higher order and chaos. Furthermore, by using the straight-line stabilization method, the chaos can be eliminated. This paper has an important theoretical and practical significance to the electricity market under the background of developing new energy.

\section{Introduction}

In 1980s, chaos theory was first introduced into the economic research. Chaotic economists used the basic mathematic theory of chaos to improve the existing models of economic phenomena. The economic system is whether a chaotic system is a hot topic in the economic field. Bifurcation theory based on difference equation has been applied in all branches of chaos [1]. In recent years, a series of dynamic game models on the output decision (Cournot model) and price decision (Bertrand model) have been studied in related references. Agiza [2] and Kopel [3] have considered bounded rationality and established duopoly Cournot model with linear cost functions. From then on, the model has been extended to multioligopolistic market. Bischi et al. [4] suppose that firms determine their output based on the reaction functions, that is, all the players take adaptive expectation. Agiza and Elsadany [5] have improved the model that contains two-types of heterogeneous players: boundedly rational player and adaptive expectation player. Zhang et al. [6] have further improved 
the model with nonlinear cost functions. Matsumoto and Nonaka [7] have researched the complexity of Cournot model with linear cost functions. Ma and Ji [8] have constructed and considered a Cournot model in electric power triopoly with nonlinear inverse demand, and the model is further studied by Ji [9] based on heterogeneous players. Ma and Feng [10] have studied the chaotic behavior in retailer's demand model. Xin et al. [11] have researched the complex dynamics of an adnascent-type game model. Chen et al. [12] have used Bertrand model with linear demand functions to study the competition in Chinese telecommunications market. Sun and Ma [13] have introduced a Bertrand model with nonlinear demand functions in Chinese cold rolled steel market and researched the complexity and the control of the model. Yassen and Agiza [14] have considered a Cournot duopoly game and the model with delayed rationality. In these pieces of literatures, adjustment speed or other parameters are taken as bifurcation parameters, and complex results such as period doubling bifurcation, unstable period orbits, and chaos are found.

Economic dynamics seem to devote new interest to delay differential equations. This is because some economic phenomena cannot be described exhaustively with pure (linear or nonlinear) differential equations. Differential equations with time delay play an important role in economy, engineering, biology, and social sciences, because a great deal of problems may be described with their help. Based on the game model [8], a new duopoly game model with delayed bounded rationality in the electricity market is obtained. The duopoly model with delayed bounded rationality is closer to the economic reality and is worth being used in oligopoly. Suppose the inverse demand function is nonlinear, and cost functions are one nonlinear and one linear. In this model, the bounded rational players regulate output speed according to marginal profit and decide the output. By theoretical analysis and numerical simulation, the stable region about the output adjustment speed parameters is derived. It is shown that the output adjustment speed lead to the chaos at a definite range. It has an important theoretical and applied significance to research the complexity of new style nonlinear dynamical system.

The paper is organized as follows. In Section 2, the dynamics of a game with delayed bounded rationality is presented. In Section 3, the existence, local stability, and bifurcation of the equilibrium points are also analyzed. Numerical simulations are used to show the complex characteristics of the system via computation of Lyapunov exponents, confirmation of the system sensitive dependence on initial conditions and calculation of the fractal dimension of the chaotic attractor. In Section 4, bifurcation and chaos control of the model is considered with the straight-line stabilization method. Finally, some conclusions are made.

\section{Model}

Suppose that there are two representative electricity enterprises (the enterprise $X$ represents the traditional coal electricity enterprise, the enterprise $Y$ represents new energy enterprise such as water electricity, nuclear power, or solar energy, or wind energy, etc.) in the electricity market, and they provide electric power to consumers through the electric utilities. The electricity enterprises $X, Y$ make the optimal output decision and suppose the $t$-output is $q_{i}(t),(i=1,2)$, respectively.

At each period $t$, the price $p$ is determined by the total output $Q(t)=q_{1}(t)+q_{2}(t)$. According to [8], the inverse demand function is

$$
p=p(Q)=m-n Q^{2}
$$


We propose the cost function of the enterprise $X$ is nonlinear. We propose the cost function of the enterprise $Y$ is linear because the new energy enterprise has lower variable cost. So, the cost functions of the two enterprises are as follows:

$$
\begin{aligned}
& V C_{X}=V C_{X}\left(q_{1}\right)=a_{1}+b_{1} q_{1}+c_{1} q_{1}^{2}, \\
& V C_{Y}=V C_{Y}\left(q_{2}\right)=a_{2}+b_{2} q_{2} .
\end{aligned}
$$

It is assumed that the wheeling rate is $\gamma$. The profits of the companies are

$$
\begin{aligned}
& \pi_{X}(t)=x(t)\left[m-n Q^{2}(t)\right]-\left(a_{1}+b_{1} q_{1}(t)+c_{1} q_{1}(t)^{2}\right)-\gamma q_{1}(t), \\
& \pi_{Y}(t)=y(t)\left[m-n Q^{2}(t)\right]-\left(a_{2}+b_{2} q_{2}(t)-r q_{2}(t)\right) .
\end{aligned}
$$

As the game between the enterprises is a continuous and long-term repeated dynamic process in the electricity market, so the dynamic adjustment of this repeated Cournot duopoly game with bounded rational players is as follows:

$$
\begin{array}{ll}
q_{1}(t+1)=q_{1}(t)+\alpha_{1} q_{1}(t) \frac{\partial \pi_{x}}{\partial q_{1}}, & 0 \leq \alpha_{1} \leq 1, \\
q_{2}(t+1)=q_{2}(t)+\alpha_{2} q_{2}(t) \frac{\partial \pi_{y}}{\partial q_{2}}, & 0 \leq \alpha_{2} \leq 1,
\end{array}
$$

where $\alpha_{i},(i=1,2)$ is output adjustment speed parameters.

Combining (2.3), (2.4), a dynamic duopoly game with bounded rationality has the following form:

$$
\begin{aligned}
& q_{1}(t+1)=q_{1}(t)+\alpha_{1} q_{1}(t)\left[-3 n q_{1}^{2}(t)-n q_{2}^{2}(t)-4 n q_{1}(t) q_{2}(t)-2 c_{1} q_{1}(t)+m-b_{1}-r\right] \\
& q_{2}(t+1)=q_{2}(t)+\alpha_{2} q_{2}(t)\left[-3 n q_{2}^{2}(t)-n q_{1}^{2}(t)-4 n q_{1}(t) q_{2}(t)+m-b_{2}-r\right] .
\end{aligned}
$$

According to [14], there are two reasons for the occurrence of delayed structure in economic models: one is that decisions made by economic agents at time $t$ is depended on the past observed variables by means of a prediction feedback, and the other is that the functional relationships describing the dynamics of the model both depend on the current state of the economy and in a nontrivial manner on past states.

Due to incomplete information and delayed decision, we propose that there is one step $(T=1)$ delay in the output of the mutual enterprises. Therefore, the dynamic game model (2.5) with delayed is as follows:

$$
\begin{aligned}
& q_{1}(t+1)=q_{1}(t)+\alpha_{1} q_{1}(t)\left[-3 n q_{1}^{2}(t)-n q_{2}^{2}(t-1)-4 n q_{1}(t) q_{2}(t-1)-2 c_{1} q_{1}(t)+m-b_{1}-r\right], \\
& q_{2}(t+1)=q_{2}(t)+\alpha_{2} q_{2}(t)\left[-3 n q_{2}^{2}(t)-n q_{1}^{2}(t-1)-4 n q_{1}(t-1) q_{2}(t)+m-b_{2}-r\right] .
\end{aligned}
$$




\section{Complex Dynamics Character of System (2.6)}

The bounded rational player makes output decision on the basis of the marginal profit of the last period. The company decides to increase output if it has a positive marginal profit and decrease output if the marginal profit is negative. Thus, output adjustment speed parameter $\alpha_{i},(i=1,2)$ has an important effect on game results. In the following section, the effect of $\alpha_{i},(i=1,2)$ on dynamical behaviors of system (2.6) will be taken into account.

\subsection{The Equilibrium Point and Stability Analysis}

The bifurcation parameters are $\alpha_{1}, \alpha_{2}$, and the other parameters of system (2.6) are as follows: $m=5.2, n=0.95, b_{1}=0.42, c_{1}=0.25, b_{2}=0.37$, and $\gamma=0.15$.

By solving the following equations, the fixed points of system (2.6) can be obtained

$$
\begin{aligned}
& q_{1}(t)\left[-3 n q_{1}^{2}(t)-n q_{2}^{2}(t-1)-4 n q_{1}(t) q_{2}(t-1)-2 c_{1} q_{1}(t)+m-b_{1}-r\right]=0, \\
& q_{2}(t)\left[-3 n q_{2}^{2}(t)-n q_{1}^{2}(t-1)-4 n q_{1}(t-1) q_{2}(t)+m-b_{2}-r\right]=0 .
\end{aligned}
$$

Equation (3.1) is solved, and three fixed points $p_{1}(0.6993,0.8363), p_{2}(0,1.28144)$, $p_{3}(18.4879,0)$ are obtained. The stability of the Nash equilibrium point $p^{*}\left(q_{1}^{*}=0.6993, q_{2}^{*}=\right.$ $0.8363)$ is only considered here. the form

To study the stability of system (2.6), we rewrite it as a fourth-dimensional system in

$$
\begin{gathered}
x(t+1)=q_{1}(t), \\
y(t+1)=q_{2}(t), \\
q_{1}(t+1)=q_{1}(t)+\alpha_{1} q_{1}(t)\left[-3 n q_{1}^{2}(t)-n y^{2}(t)-4 n q_{1}(t) y(t)-2 c_{1} q_{1}(t)+m-b_{1}-\gamma\right], \\
q_{2}(t+1)=q_{2}(t)+\alpha_{2} q_{2}(t)\left[-3 n q_{2}^{2}(t)-n x^{2}(t)-4 n x(t) q_{2}(t)+m-b_{2}-r\right] .
\end{gathered}
$$

The Jacobian matrix of (3.2) at the Nash equilibrium point $p^{*}$ is

$$
J=\left(\begin{array}{cccc}
0 & 0 & 1 & 0 \\
0 & 0 & 0 & 1 \\
0 & j_{32} & 1+j_{33} & 0 \\
j_{41} & 0 & 0 & 1+j_{44}
\end{array}\right)
$$

where

$$
\begin{gathered}
j_{32}=\alpha_{1} q_{1}^{*}\left(-2 n q_{2}^{*}-4 n q_{1}^{*}\right), \quad j_{33}=\alpha_{1} q_{1}^{*}\left(-6 n q_{1}^{*}-4 n q_{2}^{*}-2 c_{1}\right), \\
j_{41}=\alpha_{2} q_{2}^{*}\left(-2 n q_{1}^{*}-4 n q_{2}^{*}\right), \quad j_{44}=\alpha_{2} q_{2}^{*}\left(-6 n q_{2}^{*}-4 n q_{1}^{*}\right) .
\end{gathered}
$$




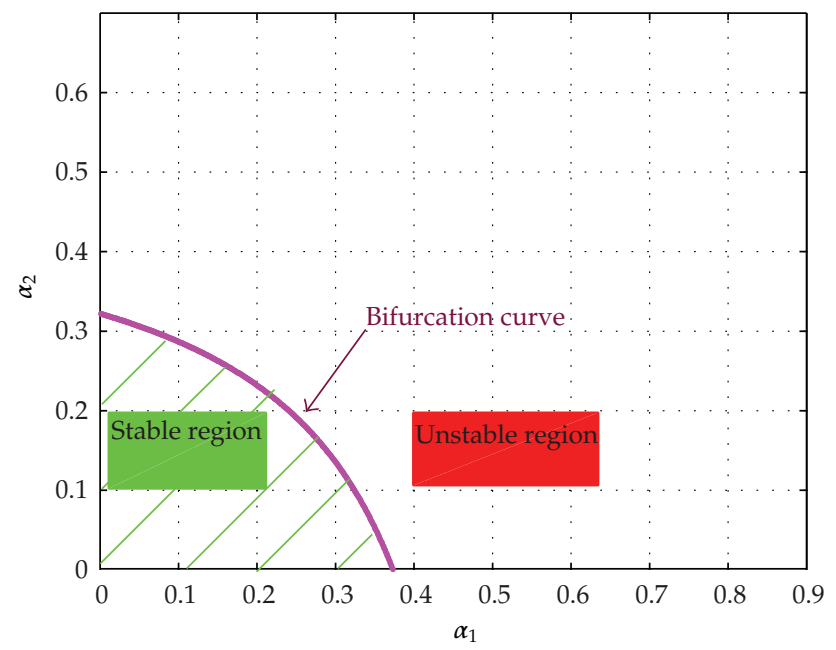

Figure 1: The stable region of Nash equilibrium point about adjustment speed $\left(\alpha_{1}, \alpha_{2}\right)$.

The characteristic polynomial of (3.2) is

$$
f(\lambda)=\lambda^{4}+A \lambda^{3}+B \lambda^{2}+C \lambda+D,
$$

where

$$
A=-\left(j_{33}+j_{44}+2\right), \quad B=\left(1+j_{33}\right)\left(1+j_{44}\right), \quad C=0, \quad D=-j_{32} j_{41} .
$$

Denote $\mu_{1}=1-D^{2}, \mu_{2}=A-C D, \mu_{3}=A-B D, \mu_{4}=C-A D, v_{1}=\mu_{4}^{2}-\mu_{1}^{2}, v_{2}=\mu_{3} \mu_{4}-\mu_{1} \mu_{2}$, and $v_{3}=\mu_{2} \mu_{4}-\mu_{1} \mu_{3}$; the necessary and sufficient conditions for the local stability of Nash equilibrium can be gained by Jury test [15]:

$$
\begin{aligned}
& \text { (i) } 1+A+B+C+D>0, \\
& \text { (ii) } 1-A+B-C+D>0, \\
& \text { (iii) }|D|<1, \\
& \text { (iv) }\left|\mu_{4}\right|<\left|\mu_{1}\right|, \\
& \text { (v) }\left|v_{3}\right|<\left|v_{1}\right| .
\end{aligned}
$$

Through computing the above equations, the local stable region of Nash equilibrium point can be obtained. The stable region of slash with positive $\left(\alpha_{1}, \alpha_{2}\right)$ is shown in Figure 1. The Nash equilibrium is stable for the values $\left(\alpha_{1}, \alpha_{2}\right)$ inside the stable region. The meaning of the stable region is that whatever initial outputs are chosen by the two electricity companies in the local stable region, they will eventually arrive at Nash equilibrium output after finite games. It is valuable to analyze the enterprises on accelerating the output adjustment speed to increase their profits. While output adjustment parameters do not change Nash equilibrium 


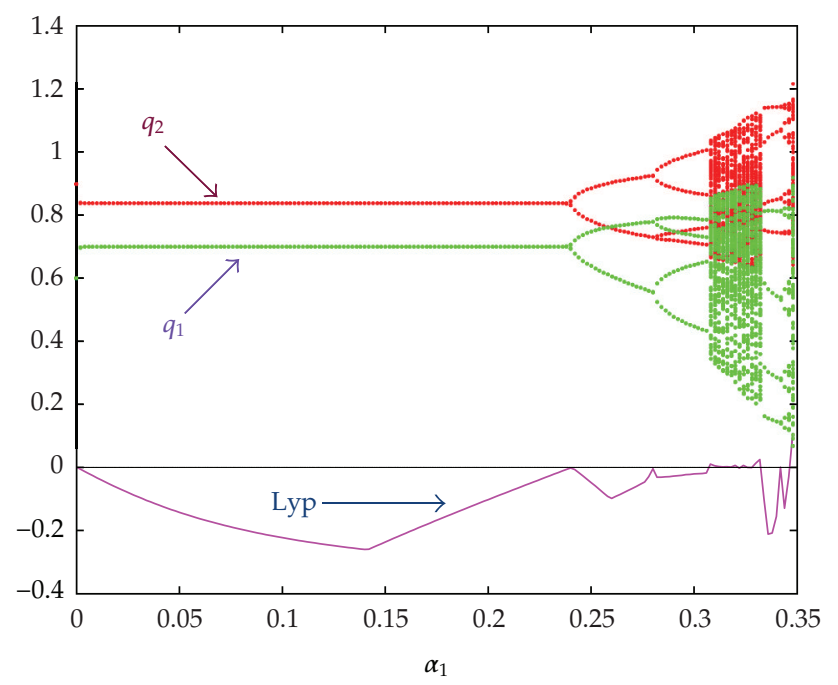

Figure 2: Bifurcation diagram and the largest Lyapunov exponent with $\alpha_{1} \in(0,0.35], \alpha_{2}=0.2$.

point, but once one party is adjusting output speed too fast and pushing $\alpha_{i},(i=1,2)$ out of the stable region, the system tends to become unstable and fall into chaos. Numerical simulation is used to analyze the characteristics of nonlinear dynamical system with the change of $\alpha_{i},(i=$ $1,2)$. Numerical results such as bifurcation diagrams, the largest Lyapunov exponent, strange attractors, sensitive dependence on initial conditions, and fractal structure will be researched.

\subsection{The Output Adjustment Speed Effect on the System}

Once company $X$ accelerates output adjustment speed and pushes $\alpha_{1}$ out of the stable region, the stability of Nash equilibrium point will change. Figure 2 demonstrates that the output evolution of the duopoly starts with equilibrium state, undergoes period doubling bifurcation to chaotic state, occurs period doubling bifurcation again and ends with chaotic state when the output adjustment speed $\alpha_{1}$ increases and $\alpha_{2}=0.2$.

When $\alpha_{2}=0.2$, the diagrams of bifurcation and the largest Lyapunov exponent with $\alpha_{1}$ increasing are shown in Figure 2. For $0<\alpha_{1}<0.2362$, the largest Lyapunov exponent is negative and the system (2.6) is stable at Nash equilibrium point. For $0.2362<\alpha_{1}<0.2790$, the largest Lyapunov exponent is negative and system (2.6) has an orbit of 2-cycle. For $0.2790<\alpha_{1}<0.3059$ and $0.3329<\alpha_{1}<0.3427$, the largest Lyapunov exponent is negative and system (2.6) has an orbit of 4-cycle. For $0.3427<\alpha_{1}<0.3476$, the largest Lyapunov exponent is negative and system (2.6) has an orbit of 8-cycle. For $0.3059<\alpha_{1}<0.3280$, the largest Lyapunov exponent just a little bigger than zero and system (2.6) has a double chaotic attractor as shown in Figures 3(a) and 3(b). For $0.3280<\alpha_{1}<0.3329$ and $0.3476<\alpha_{1}<0.35$, the largest Lyapunov exponent is obviously positive and system (2.6) is in a chaotic state as shown in Figures 3(c) and 3(d).

Similarly, Figure 4 shows a one-parameter bifurcation diagram and the largest Lyapunov exponent with respect to $\alpha_{2}$ and when $\alpha_{1}=0.15$. We can see that Nash equilibrium point is stable for $0<\alpha_{2}<0.2621$, which implies that output of two firms is in equilibrium state. With $\alpha_{2}$ increasing, the stability of equilibrium point changes, and output undergoes 


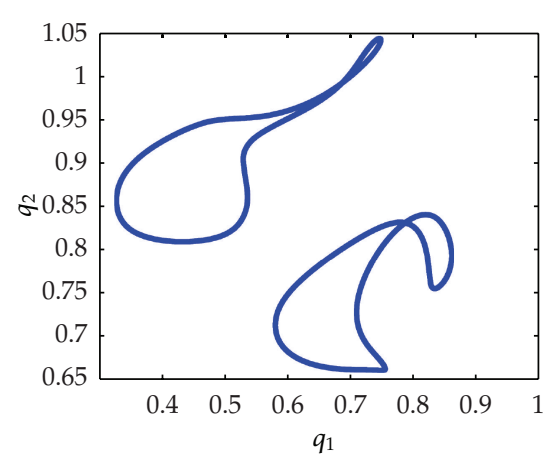

(a)

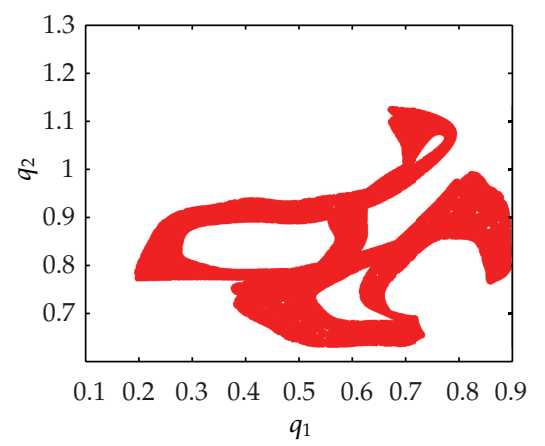

(c)

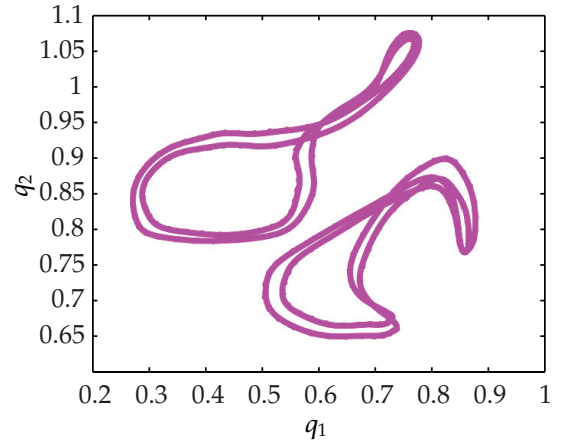

(b)

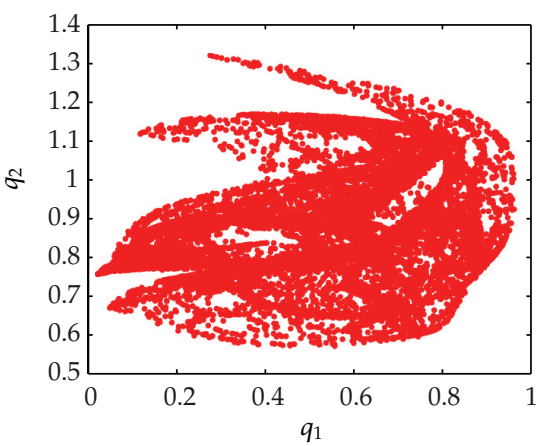

(d)

Figure 3: The typical dynamical behaviors of system (2.6) with $\alpha_{2}=0.2$ and (a) $\alpha_{1}=0.31$; (b) $\alpha_{2}=0.32$; (c) $\alpha_{1}=0.33 ;$ (d) $\alpha_{1}=0.348$.

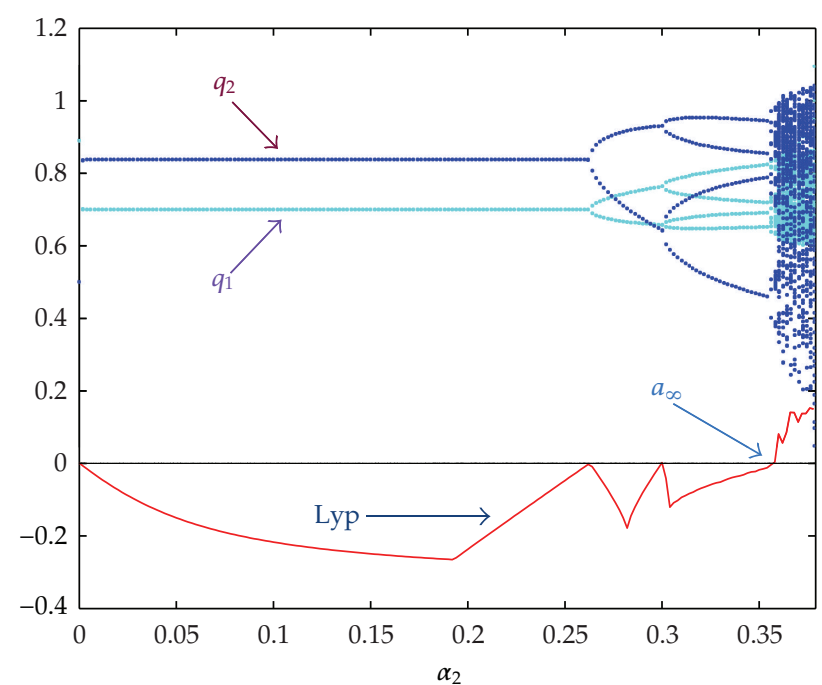

Figure 4: Bifurcation diagram and the largest Lyapunov exponent with $\alpha_{2} \in(0,0.379], \alpha_{1}=0.15$. 


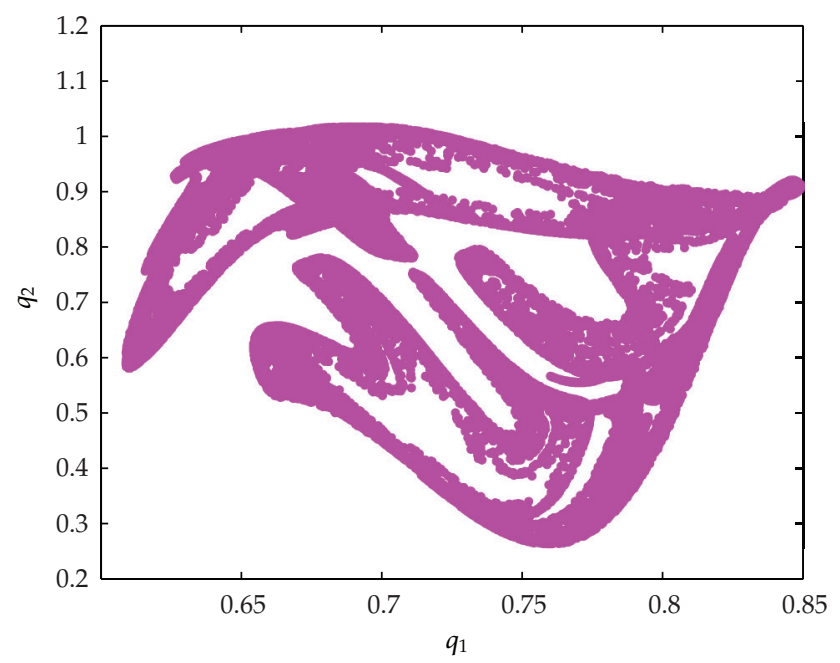

Figure 5: Chaos attractor of system (2.6) with $\alpha_{2}=0.362, \alpha_{1}=0.15$, and initial point $\left(q_{1}^{0}=0.6, q_{2}^{0}=0.5\right)$.

doubling period bifurcation and eventually falls into chaos. $\alpha_{2} \in(0.2621,0.3]$ is the range of 2-cycle output fluctuation. For $\alpha_{2}>0.3$, output doubling occurs again. $\alpha_{2} \in(0.3,0.3537]$ is the range of 4-cycle output fluctuation. $\alpha_{2} \in(0.3537,0.379]$ is the range of output falling into chaos.

Figure 5 illustrates chaos attractor with initial point $\left(q_{1}^{0}=0.6, q_{2}^{0}=0.5\right)$ when $\alpha_{2}=$ $0.362, \alpha_{1}=0.15$.

The sensitive dependence on initial conditions is one of the important features of chaos. To verify whether system (2.6) depends on initial values sensitively, the relationships between output and time are shown in Figures 6(a), 6(b), 6(c), and 6(d) when $\alpha_{1}=0.348, \alpha_{2}=$ 0.2 and $\alpha_{1}=0.15, \alpha_{2}=0.362$, respectively. At first, the difference is indistinguishable, but with the number of the game increasing, the difference between them is huge. This demonstrates that only a little difference between initial data will have a great impact on the results of the game. It further proves that system (2.6) falls into a chaotic state when $\alpha_{1}=0.348, \alpha_{2}=0.2$ and $\alpha_{1}=0.15, \alpha_{2}=0.362$. While the system is in a chaotic state, the market becomes volatile and it is difficult for electricity companies to plan long-term strategy. A slight adjustment of the initial output can have a great effect on the game results.

A fractal dimension is taken as a criterion to judge whether the system is chaotic. There are many specific definitions of fractal dimension and none of them should be treated as the universal one. According to [16], we adopt the following definition of fractal dimension:

$$
d=j-\frac{\sum_{1}^{j} \lambda_{i}}{\lambda_{j+1}}
$$

where $\lambda_{1}>\lambda_{2}>, \ldots, \lambda_{n}$ are the Lyapunov exponents and $j$ is the maximum integer for which $\sum_{1}^{j} \lambda_{i}>0$ and $\sum_{1}^{j+1} \lambda_{i}<0$. If $\lambda_{i} \geq 0, i=1,2, \ldots, n$, then $d=n$. If $\lambda_{i}<0, i=1,2, \ldots, n$, then $d=0$.

The Lyapunov exponents of system (2.6) are $\lambda_{1}=0.067051, \lambda_{2}=-0.106206$ when $\alpha_{1}=0.15, \alpha_{2}=0.362$. The largest Lyapunov exponent $\lambda_{1}$ is positive, which indicates system 


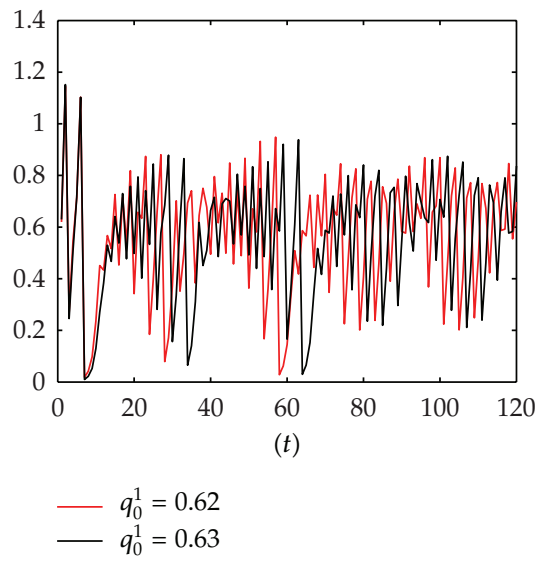

(a)

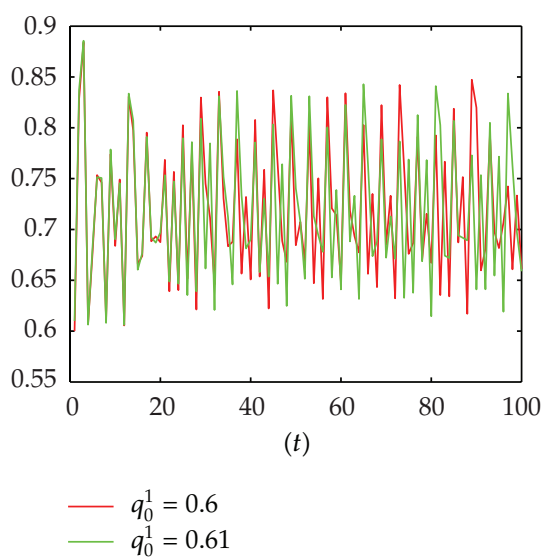

(c)

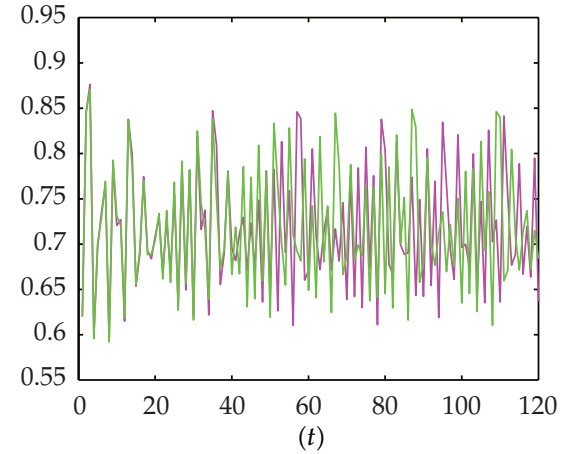

$q_{0}^{2}=0.52$

$q_{0}^{2}=0.53$

(b)

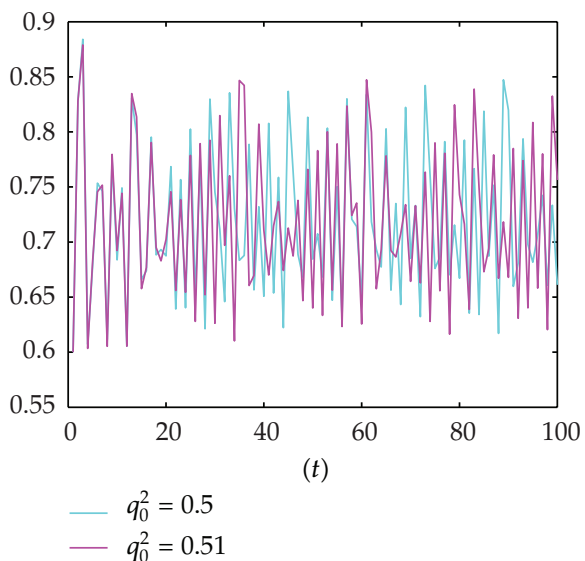

(d)

Figure 6: Sensitive dependence on initial conditions: the two orbits of (a) $q_{1}$-coordinates for initial points are $(0.62,0.52)$ and $(0.63,0.52)$; (b) $q_{2}$-coordinates for initial points are $(0.62,0.52)$ and $(0.62,0.53)$; (c) $q_{1^{-}}$coordinates for initial points are $(0.6,0.5)$ and $(0.61,0.5) ;(d) q_{2}$-coordinates for initial points are $(0.6,0.5)$ and $(0.6,0.51)$.

(2.6) is in a chaotic state. Fractal dimension illustrates that the chaotic motion has self-similar structure, that is to say, the chaotic motion follows a definite rule. This is an important difference between chaotic motion and stochastic motion. The fractal dimension of system (2.6) is $d=2-\lambda_{1} / \lambda_{2} \approx 1.6313$. The fractal dimension reflects the space density of the chaotic attractor [17]. The larger the dimension of the chaotic attractor is, the larger is the occupied space. Consequently, the structure is more compact, and the system is more complicated and vice versa. The fractal dimension of the $2 \mathrm{D}$ discrete system (2.6) is more than 1.5 , so the occupied space is big and the structure is tight, which can be seen in Figure 5.

\section{Chaos Control}

The numerical simulation results show that the oligopolistic market becomes unstable and even falls into chaos when output adjustment speed parameter goes beyond the stable region. 
All the players will be harmed and the market will become irregular when chaos occurs. So, nobody is able to make good strategies and decide reasonable output. To avert the risk, it is a good ideal for duopoly to maintain at Nash equilibrium output.

Perturbation feedback and nonfeedback are two methods for the chaos control. Recently, Yang et al. [18] and Xu et al. [19] proposed a new control method, which is called the straight-line stabilization method. This method is adopted to control the chaos in this paper

$$
\begin{aligned}
\delta=\left(\begin{array}{l}
\delta_{1} \\
\delta_{2}
\end{array}\right)= & \left(\begin{array}{cc}
k-\left(1+j_{33}\right) & 0 \\
0 & k-\left(1+j_{44}\right)
\end{array}\right)\left(\begin{array}{l}
q_{1}(t)-q_{1}^{*} \\
q_{2}(t)-q_{2}^{*}
\end{array}\right) \\
& -\left(\begin{array}{cc}
0 & j_{32} \\
j_{41} & 0
\end{array}\right)\left(\begin{array}{l}
q_{1}(t-1)-q_{1}^{*} \\
q_{2}(t-1)-q_{2}^{*}
\end{array}\right) \\
= & \left(\begin{array}{c}
{\left[k-\left(1+j_{33}\right)\right]\left(q_{1}(t)-q_{1}^{*}\right)-j_{32}\left(q_{2}(t-1)-q_{2}^{*}\right)} \\
-j_{41}\left(q_{1}(t-1)-q_{1}^{*}\right)+\left[k-\left(1+j_{44}\right)\right]\left(q_{2}(t)-q_{2}^{*}\right)
\end{array}\right),
\end{aligned}
$$

where $|k|<1$ is the feedback control parameter and other parameters are the same as above.

Adding the external control signal (4.1) to system (2.6), the controlled system is as follows:

$$
\begin{aligned}
q_{1}(t+1)=q_{1}(t)+\alpha_{1} q_{1}(t)[ & -3 n q_{1}^{2}(t)-n q_{2}^{2}(t-1) \\
& \left.-4 n q_{1}(t) q_{2}(t-1)-2 c_{1} q_{1}(t)+m-b_{1}-\gamma\right]+\delta_{1}, \\
q_{2}(t+1)=q_{2}(t)+\alpha_{2} q_{2}(t)[ & -3 n q_{2}^{2}(t)-n q_{1}^{2}(t-1) \\
& \left.-4 n q_{1}(t-1) q_{2}(t)+m-b_{2}-\gamma\right]+\delta_{2} .
\end{aligned}
$$

It can be seen from Figure 7, at $\left(\alpha_{1}=0.15, \alpha_{2}=0.362\right)$, that controlled system (4.2) stabilized at Nash equilibrium point when $-0.9876<k<-0.28$. It demonstrates that the stable control of the specific goal can be realized even if the perturbation is very small.

At $k=-0.5, \alpha_{1}=0.15$, the stable range of system (4.2) is $0.289<\alpha_{2}<0.436$. The range of 2-cycle bifurcation is $0.436<\alpha_{2}<0.5171$. The range of 4-cycle bifurcation is $0.5171<\alpha_{2}<$ 0.5374 . For $0.5374<\alpha_{2}<0.6084$, system (4.2) is in the chaotic state. The details are shown in Figure 8, which indicates that once system (2.6) is under a chaotic state, system (4.2) can be efficient to eliminate chaos.

Figure 9 shows chaos attractor of the controlled system (4.2) with initial point $q_{1}^{0}=$ $0.6, q_{2}^{0}=0.5$ and $\alpha_{1}=0.15, \quad \alpha_{2}=0.56, k=-0.5$.

\section{Conclusions}

A new dynamics of nonlinear duopoly game in the electricity market with delayed bounded rationality is established in this paper. The stability of equilibria, bifurcation, and chaotic behavior of the duopoly game are investigated. It is found that bifurcation, chaos, and other complex phenomena occur when the output adjustment speed parameter changes. It is well 


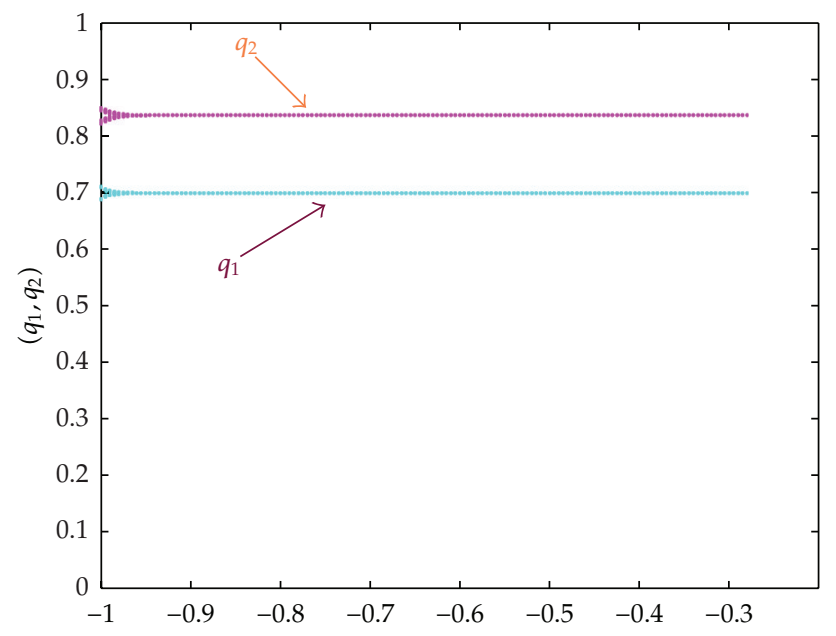

$(k)$

Figure 7: Bifurcation diagram with $\alpha_{1}=0.15, \alpha_{2}=0.362, k \in[-1,-0.2]$.

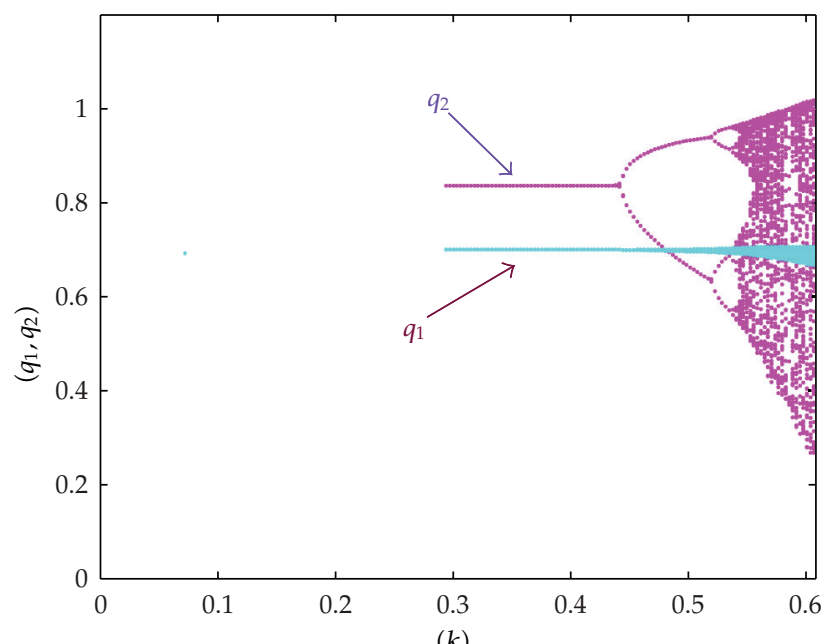

$(k)$

Figure 8: Bifurcation diagram with $\alpha_{1}=0.15, k=-0.5$, and $\alpha_{2} \in[0,0.6084]$.

known that the occurrence of chaos depends on the values of bifurcation parameters. The straight-line stabilization method is used to control the period-doubling bifurcation, unstable periodic orbits, and chaos. The system quickly arrived at the Nash equilibrium point when a small perturbation is applied in the chaos region. The research results have an important theoretical and practical significance to the electricity market under the view of developing new energy. This paper also shows guidance for electricity companies to formulate strategies of output and is helpful for the government to formulate relevant policies to macrocontrol economy. 


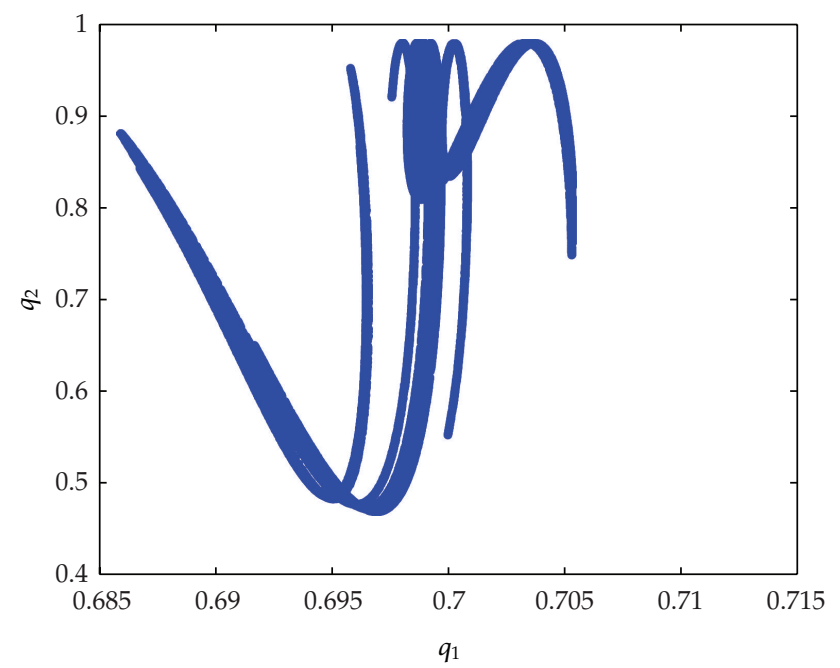

Figure 9: Chaos attractors of the controlled system (4.2) with $\alpha_{1}=0.15, \alpha_{2}=0.56, k=-0.5$, and initial point $q_{1}^{0}=0.6, q_{2}^{0}=0.5$.

\section{Acknowledgments}

This work was supported by the Doctoral Scientific Fund Project of the Ministry of Education of China under Contract no. 20090032110031 and was also supported by the National Natural Science Foundation of China under Contract no. 61273231.

\section{References}

[1] G. Zou and F. Gao, "Economics investigation and chaos theory," Journal of Quantum Technologies and Economics, vol. 4, pp. 61-68, 1991.

[2] H. N. Agiza, "On the analysis of stability, bifurcation, chaos and chaos control of Kopel map," Chaos, Solitons and Fractals, vol. 10, no. 11, pp. 1909-1916, 1999.

[3] M. Kopel, "Simple and complex adjustment dynamics in Cournot duopoly models," Chaos, Solitons and Fractals, vol. 7, no. 12, pp. 2031-2048, 1996.

[4] G. I. Bischi, C. Mammana, and L. Gardini, "Multistability and cyclic attractors in duopoly games," Chaos, Solitons E Fractals, vol. 11, no. 4, pp. 543-564, 2000.

[5] H. N. Agiza and A. A. Elsadany, "Chaotic dynamics in nonlinear duopoly game with heterogeneous players," Applied Mathematics and Computation, vol. 149, no. 3, pp. 843-860, 2004.

[6] J. Zhang, Q. Da, and Y. Wang, "Analysis of nonlinear duopoly game with heterogeneous players," Economic Modelling, vol. 24, no. 1, pp. 138-148, 2007.

[7] A. Matsumoto and Y. Nonaka, "Statistical dynamics in a chaotic Cournot model with complementary goods," Journal of Economic Behavior and Organization, vol. 61, no. 4, pp. 769-783, 2006.

[8] J. H. Ma and W. Z. Ji, "Complexity of repeated game model in electric power triopoly," Chaos, Solitons and Fractals, vol. 40, no. 4, pp. 1735-1740, 2009.

[9] W. Ji, "Chaos and control of game model based on heterogeneous expectations in electricpower triopoly," Discrete Dynamics in Nature and Society, vol. 2009, 8 pages, 2009.

[10] J. Ma and Y. Feng, "The study of the chaotic behavior in retailer's demand model," Discrete Dynamics in Nature and Society, Article ID 792031, 12 pages, 2008.

[11] B. Xin, J. Ma, and Q. Gao, "Complex dynamics of an adnascent-type game model," Discrete Dynamics in Nature and Society, vol. 2008, Article ID 467972, 12 pages, 2008.

[12] F. Chen, J. H. Ma, and X. Q. Chen, "The study of dynamic process of the triopoly games in chinese 3G telecommunication market," Chaos, Solitons and Fractals, vol. 42, no. 3, pp. 1542-1551, 2009. 
[13] Z. Sun and J. Ma, "Complexity of triopoly price game in Chinese cold rolled steel market," Nonlinear Dynamics, vol. 67, no. 3, pp. 2001-2008, 2012.

[14] M. T. Yassen and H. N. Agiza, "Analysis of a duopoly game with delayed bounded rationality," Applied Mathematics and Computation, vol. 138, no. 2-3, pp. 387-402, 2003.

[15] L. Edelstein-Kashet, Mathematical Models in Biology, Random House, New York, NY, USA, 1992.

[16] J. L. Kaplan and J. A. Yorke, "Preturbulence: a regime observed in a fluid flow model of Lorenz," Communications in Mathematical Physics, vol. 67, no. 2, pp. 93-108, 1979.

[17] B. Liu and J. Peng, Nonlinear Dynamics, Higher Education Press, Beijing, China, 2004.

[18] L. Yang, Z. Liu, and J. M. Mao, "Controlling hyperchaos," Physical Review Letters, vol. 84, no. 1, pp. 67-70, 2000

[19] H. Xu, G. Wang, and S. Chen, "Controlling chaos by a modified straight-line stabilization method," European Physical Journal B, vol. 22, no. 1, pp. 65-69, 2001. 


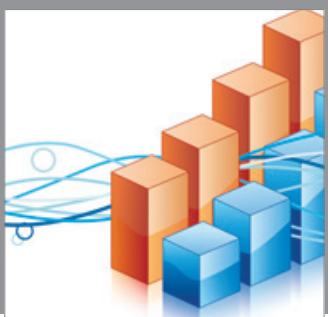

Advances in

Operations Research

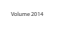

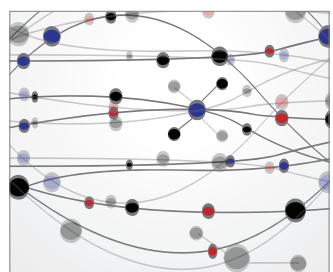

\section{The Scientific} World Journal
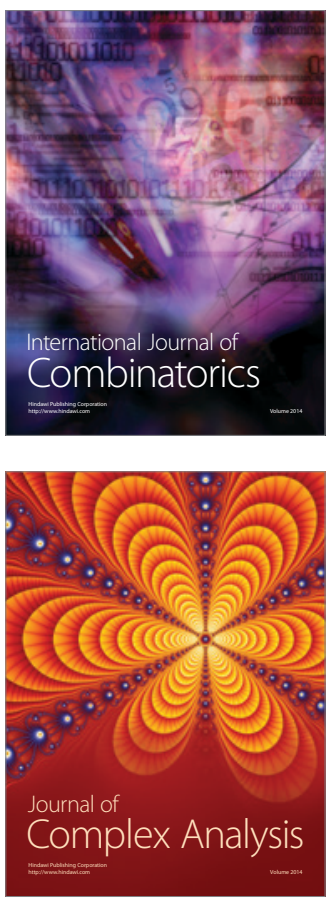

International Journal of

Mathematics and

Mathematical

Sciences
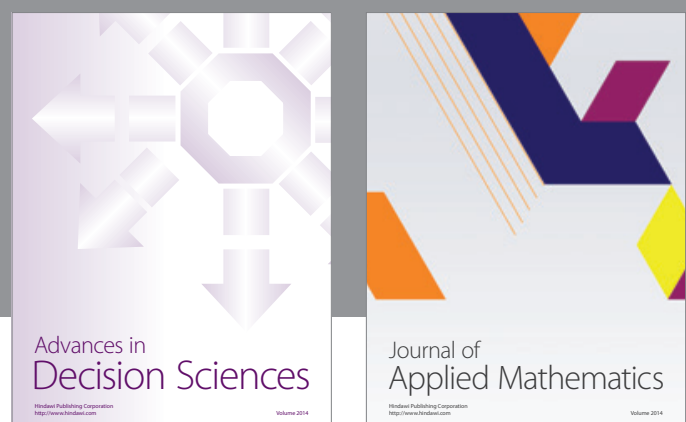

Journal of

Applied Mathematics
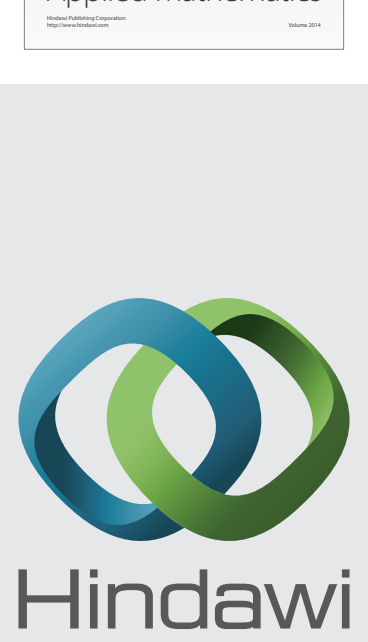

Submit your manuscripts at http://www.hindawi.com
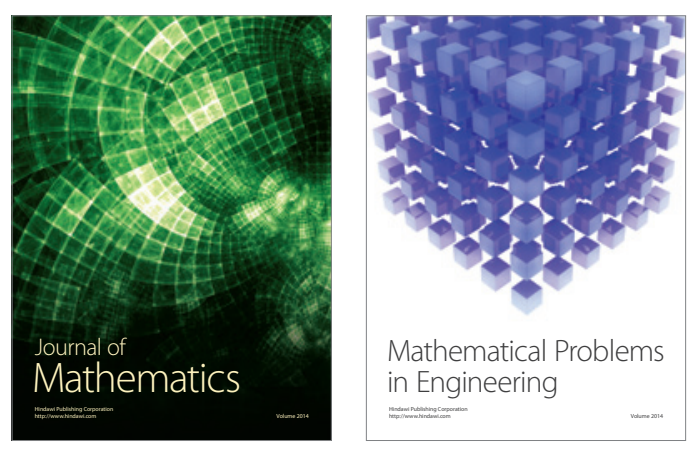

Mathematical Problems in Engineering
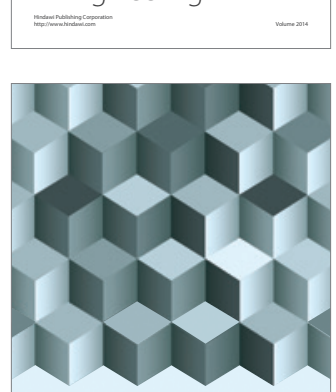

Journal of

Function Spaces
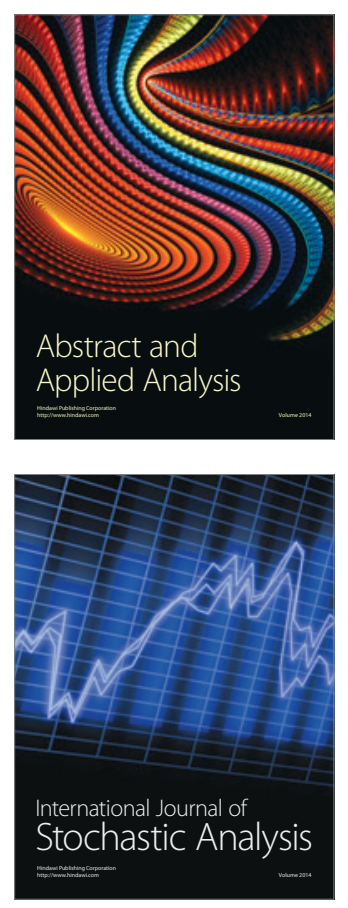

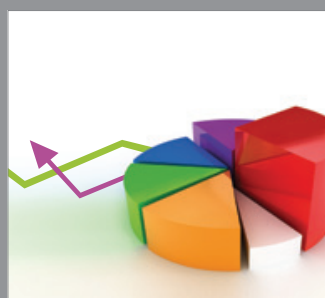

ournal of

Probability and Statistics

Promensencen
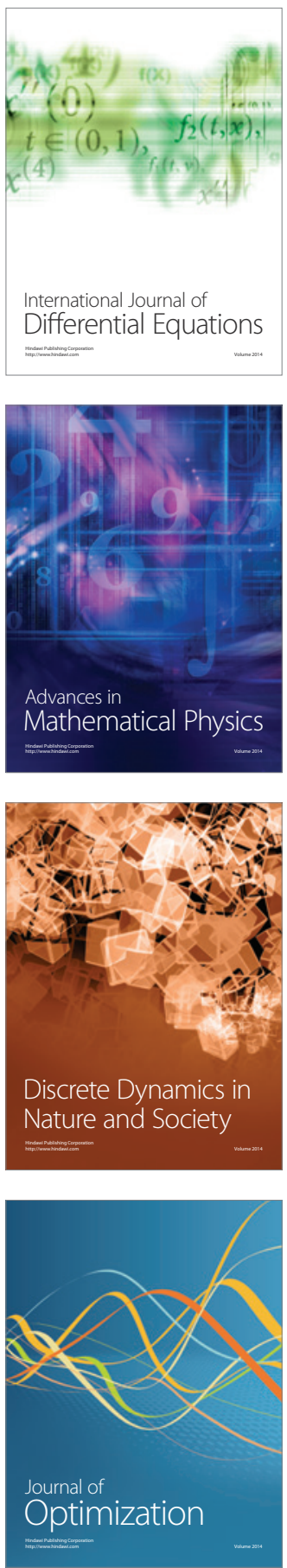